\title{
WORLD
}

RES OURCES

WORKING PAPER

\author{
I N S T I T UT E
}

\section{APPLYING CLIMATE SERVICES TO TRANSFORMATIVE ADAPTATION IN AGRICULTURE}

LAURIE ASHLEY, REBECCA CARTER, TYLER FERDINAND, RICHARD CHOULARTON, NAMBI APPADURAI, NAMRATA GINOYA, PARVATHI PREETHAN

\section{EXECUTIVE SUMMARY}

\section{Highlights}

- Climate change is already pushing some natural and human systems to their limits. Increasingly severe impacts on agricultural systems will require transformative adaptation-that is, significant changes to fundamental aspects of agricultural systems in response to or anticipation of longer-term climate-change impacts.

- Climate services (CS) have generated sophisticated knowledge about climate change and its impacts on agricultural production across timescales, but they could be enhanced to support transformative adaptation. Enhanced CS would be designed specifically to help higher-level decision-makers like adaptation funders, governments, and development planners understand where, when, and what kind of transformative adaptation measures will be needed and guide long-term climate resilience.

- Enhanced CS would include information to help adaptation planners, funders, and practitioners better apply medium- and longer-term climate-change projections, combined with crop models and expert analysis, to gain insights into where and when existing agricultural systems may no longer be viable, assess more resilient options, and map pathways for change.

- Better climate information will be inadequate unless it is integrated into adaptation and development planning, policy, and investments. This requires the inclusive, participatory development of sustainable platforms that align climate research, development, and applications with policy and planning processes.

\section{CONTENTS}

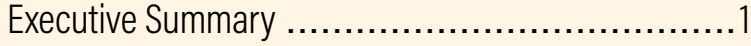

Introduction ..................................... 4

Enhancing and Applying CS to Advance

Transformative Adaptation ............................. 8

Recommendations ...................................17

Appendix A. Leveraging Crop-Climate Models

and Platforms for Transformative Adaptation ......... 19

Appendix B. Inclusion of Transformative Adaptation

in NAPs, NDCs, and KJWA Submissions 20

Appendix C. Stages of Applying CS to Transformative

Adaptation and Questions for Planning .............. 21

References ......................................... 23

Acknowledgments..................................26

About the Authors ................................. 26

About WRI ...........................................26

Working Papers contain preliminary research, analysis, findings, and recommendations. They are circulated to stimulate timely discussion and critical feedback, and to influence ongoing debate on emerging issues. Working papers may eventually be published in another form and their content may be revised.

Suggested Citation: Ashley, L., R. Carter, T. Ferdinand, et al. 2020. "Applying Climate Services to Transformative Adaptation in Agriculture" Working Paper. Washington, DC: World Resources Institute. Available online at www.wri.org/publication/applyingclimate-services. 


\section{Background}

As per Carter et al. (2018), this paper defines transformative adaptation for agriculture as intentional alterations that are so significant that they change fundamental aspects of agricultural production systems in response to or in anticipation of climate impacts. Such alterations are generally at broad scale and often include one or more of the following attributes:

Shifting the geographical locations where specific types of crops and livestock are produced and the systems that support them are located; and/or

- Applying new methodologies and technologies that substantially change the types of agricultural products or the way existing ones are produced within a particular region or production system; and/or

- Fundamentally altering a region's predominant type of agricultural landscape-for example, from cropping to aquaculture-as the result of changes to multiple aspects of food production systems and/or supply chains.

Transformative approaches offer the potential to reduce crisis and conflict, avoid maladaptation, and ensure that adaptation investments made today will prove strategic further into the future (Carter et al. 2018). This paper explores how CS-systems for developing and providing climate information to meet users' needs (WMO 2018)could be enhanced and applied to advance transformative adaptation in agriculture where and when it is needed.

\section{Key Findings}

Despite the increasingly urgent need to significantly shift agricultural systems to achieve longterm resilience, strategies to do so are rarely included in adaptation and development plans. A review of 196 Nationally Determined Contributions (NDCs), the commitments that countries made to climate action under the UNFCCC Paris Agreement, 9 National Adaptation Plans (NAPs), and 48 submissions to the UNFCCC Koronivia Joint Work on Agriculture (KJWA) platform shows that only 7 (or 2.7 percent) of these 253 documents describe applications of $\mathrm{CS}$ for adaptation planning that could be considered transformative; for example, by explicitly noting the use of long-term climate projections (see Appendix B). Yet an increasing number of agriculture system shifts will need targeted data from CS to phase in the significant, systemic changes that will be required to keep up with intensifying climate-change impacts.
To guide long-term climate resilience in agricultural production systems, CS must increase focus on decadal and longer-term climate-impact analysis within planning horizons out to around 2050. Existing CS have provided impressive benefits, including cost-benefit ratios ranging from 1:2 to 1:10 for investments in national meteorological services. Evidence suggests that using CS in agricultural decision-making resulted in gains of up to 66 percent in yields or income (Vaughan et al. 2017). To date, CS for agricultural adaptation have largely focused on daily weather and seasonal climate forecasts, which are critical for farmers to make day-to-day management decisions. However, planning for and implementing broader, longer-term interventions will require climate data that provide insights further into the future and should be combined with ongoing efforts to improve the shorter-term CS critical for daily and seasonal decision-making. Improved long-term (e.g., 2050) and decadal climate change projections must be coupled with information that illustrates which types of agricultural systems and the crop and livestock varieties within them can be expected to gain or lose productivity. Temperature and precipitation projections, for example, can be analyzed alongside crop models to gauge shifts in productivity over time.

To advance systemic change, CS must more effectively integrate information beyond agriculture, such as data on biological, environmental, economic, market, and social factors; and the use of climate information must be mainstreamed beyond planning for agriculture. For example, relocating agricultural systems, employing large-scale technology like irrigation, or shifting production type (e.g., cropping to aquaculture) will have significant implications on water supply and demand. Water is critical for all economic sectors, as well as environmental sustainability. Therefore, CS for agriculture must consider potentially competing water, land-use, and other demands from other sectors. CS that integrates information beyond agriculture and is used for more comprehensive planning can help to reduce inter-sectoral and inter-scalar competition and conflict. This will require investments in modeling, projections, and geospatial data that are downscaled, crosssectoral, and long-term, including economic feasibility and cost-benefit analysis, as well as coordination among many different government agencies and other groups that produce relevant data. 
Enhanced CS need to be tailored to meet the needs of adaptation entities (e.g., policymakers, planners, funders, and practitioners) to assess the longer-term viability of existing agricultural production systems and their alternatives. For example, linking climate-change projections with agro-climatological and other applied analyses would make it easier for key decision-makers to identify agricultural systems that may be approaching viability thresholds wherein climate impacts will become so severe that these systems will no longer be feasible, despite continued investment in system maintenance (although uncertainty will remain). Such analyses would enable decision-makers to better support the development of transformative adaptation pathways, that is, coordinated sequences of short- and medium-term actions or projects that can be phased in gradually to shift agricultural production systems, with sufficient flexibility to respond to new information (e.g., new information related to improved model skill or changes in global GHG emissions) (Carter et al. 2018).

In addition to enhanced CS, adaptation entities need additional support-tools, guidance, capacity and networks-to analyze and embed such information into plans, policies, and proposals. Although analytical systems that incorporate agricultural viability information are emerging, they are often overly complex for nonexpert users, which limits their use in adaptation planning processes. Government and other planners face additional challenges in putting decadal and multidecadal projections to use, such as issues of uncertainty, risk aversion, and the ability to justify investment based on projected risk (Singh et al. 2018). Agribusinesses are leading the way in using longer-term information to guide their investments. For example, coffee and cacao producers have mapped out which areas are likely to become more or less favorable for these crops and are investing or divesting accordingly. In contrast, less is being done to tailor CS to the needs of national-level agricultural planners and policymakers or those of bilateral and multilateral funding entities. The development of CS to support transformative adaptation must be driven by user groups, based on their needs and capacity and embedded within planning processes. Without access to high-quality, tailored CS on par with those being developed to meet the seasonal needs of farmers and by global agribusinesses, developing-country governments risk being left behind in active planning, and climate-induced inequalities will continue to widen (Islam and Winkel 2017).

\section{About This Working Paper}

The Transforming Agriculture for Climate Resilience (TACR) project, funded by the Bill and Melinda Gates Foundation, aims to increase finance for agricultural adaptation and strengthen understanding of, and action and support for, transformative approaches where and when they are needed. It seeks to assist adaptationfunding entities, planners, policymakers, and practitioners in integrating transformative adaptation into planning processes, projects, and financing.

This paper was developed by considering how transformative adaptation as outlined in Carter et al. (2018) could be accelerated by enhancing the production and application of CS. The research included an extensive review of the published academic literature on the topic and an analysis of terms related to climate services in national adaptation documents and submissions to the KJWA. Consultations with the working paper's key audiences (adaptationfunding entities, planners, policymakers, and implementing agencies) at international meetings and with government officials and technical experts in Ethiopia and India also contributed to the technical and conceptual content presented here.

As with other TACR publications, this paper deliberately focuses on top-down processes and does not cover ground-up efforts, even though aspects of transformative adaptation are sometimes implemented autonomously by farmers. This is because the farmers who are able to make changes of the type we define as transformative without external support tend to be those with greater access to resources (land, credit, technical capacity, etc.) and a higher tolerance for risk. By targeting the research at adaptation-funding entities (e.g., bilateral and multilateral funders), government entities (planners, policymakers, and meteorological services), and implementing actors (e.g., nongovernmental organizations [NGOs], international governmental organizations [IGOs], private-sector companies), along with research and support agencies, our aim is to encourage them to create policies and programs that enable the poorest and most vulnerable farmers to engage in transformative adaptation where and when it is needed, with a specific focus on medium- and long-term impacts. This paper takes the approach that CS for agricultural transformation must consider local and regional environmental and socioeconomic conditions and the adaptation options and barriers linked to local contexts (IPCC 2019). 
In its next stage, the project will synthesize the framework with the key findings and recommendations of three additional technical papers that apply the framework to crop research and development, livestock production, and water management. The result will be a call to action to accelerate transformative adaptation, to be released later in 2020.

\section{INTRODUCTION}

As climate change increasingly affects agriculture around the world, reliable, timely, and targeted information about weather and climate conditions is becoming an ever more urgent requirement for adaptation decision-making. Climate information can help decision-makers understand and plan for climate risks across regions and time scales. CS are systems for developing and providing climate information to meet users' needs and assist individual and organizational decision making (WMO 2018). Existing CS address climate information needs for agricultural production over daily, seasonal, and long-term time frames, with much of the focus to date on daily to seasonal forecasts, warnings, and advisories that meet farmers' needs to better manage risk and build climate resilience (Hansen et al. 2019). CS products for agriculture continue to expand, and researchers are developing increasingly comprehensive climate and Earth systems models to improve climate information across timescales (WMO 2014e).

Yet, some existing agricultural production systems are becoming less viable due to climate-change impacts. Others face foreseeable, albeit uncertain, tipping points that will rapidly undermine crop and livestock productivity. Adapting to such significant changes in the most affected agricultural regions will require climate information that aids in decision-making regarding which crop and livestock systems (including along their value chains; e.g., processing and markets) are likely to best maintain food security and improve livelihoods over the coming decades.

Despite the growing urgency to identify, finance, and begin implementing transformative solutions to agricultural adaptation challenges, there are few documented examples of this. A review of 196 NDCs (the commitments that countries made to climate action under the UNFCCC Paris Agreement) 9 NAPs, and 48 submissions to the UNFCCC KJWA platform shows that only 7 (or 2.7 percent) of these 253 documents describe applications of CS for adaptation planning that could be considered transformative, for example by explicitly noting the use of long-term climate projections. Reasons for such omis- sions are not entirely clear, but discussions with country representatives suggest that such information is often perceived to be too complex and uncertain to include in the already protracted and challenging process of completing a NAP that includes robust planning for current and short-term climate impacts. While models and tools do exist to identify the types of information suggested in this paper, they are largely unused. Fostering greater integration of long-term climate information and planning would likely require, among other things, building more capacity, allowing for additional time in NAP processes, providing more explicit guidance, and creating more user-friendly tools.

With over 90 NAPs currently being drafted, the time is right for more specific guidance and greater investments in this area. Transformative approaches to adaptation are also thinly represented in the initial NDCs. Only 3 of over 190 submissions included language related to transformative approaches via CS. With NDCs set to be updated and strengthened in 2020, now is also a critical time to illustrate how longer-term, systemic adaptation goals can be added.

In contrast to incremental adaptation, the goal of which is to preserve existing production systems despite intensifying climate-change impacts, transformative adaptation recognizes that in some cases, fundamental aspects of agricultural production systems, such as what, where, and how food is produced, will have to change. Although incremental adaptation measures are essential and must be scaled rapidly, they can be more strategically planned and sequenced to create pathways toward transformative adaptation. This longer-term, systemic approach to adaptation offers the opportunity to avoid shortsighted investments that lead to maladaptation and improves the odds of avoiding repeated cycles of crisis and collapse (Carter et al. 2018).

Constructing transformative pathways to meet this challenge will necessarily rely on high-quality short-, medium-, and long-term climate information to ensure that shifts in agricultural practices, approaches, and locations are productive and resilient under current and future climate conditions. Adaptation planners, policymakers, implementing entities, and other stakeholders will need to use CS to anticipate which areas are likely to need transformative adaptation approaches, evaluate options for alternative systems, and map out timelines for sequencing interventions. Financial and insurance providers will need more accurate risk-assessment tools. Ultimately, this 
work must inform the actions of farmers, particularly the estimated 475 million small-scale farmers (those farming two hectares or less) in developing countries that are highly vulnerable to climate change (FAO 2016). These farmers often depend on highly climate-sensitive rain-fed production and insecure land tenure and often lack access to high-quality inputs, insurance, and inclusive financial products (FAO 2016; IPCC 2019).

Climate information over multi-decadal time frames, out to around 2050, is critical to understanding where and when agricultural systems may reach tipping points that call for transformative adaptation responses. While the speed and scale of systemic shifts are context-specific, coasts, areas reliant on glacial melt, and arid and semiarid regions that face water demands that will soon exceed dwindling supplies are likely to be among the first to experience tipping points beyond which existing agricultural production may no longer be viable. For example, places that depend on irrigation water from rapidly melting glaciers will soon face permanent as well as seasonal shortages, and some coastal areas are already encountering inundation of agricultural land and salinization of aquifers as sea level rises.

To better support significantly shifting agricultural systems to achieve longer-term resilience, CS must be enhanced to better inform more widespread or extensive changes, such as expanding irrigation and water storage, improving soil management and agroforestry practices, modifying crop or livestock variety selection, shifting cropping patterns, or applying new technologies or risk-management options (e.g., early warning systems, insurance, and financial services) (Bryan et al. 2009; IPCC 2019; Singh et al. 2018; Braimoh et al. 2016; Vaughan et al. 2017). Where agricultural transformations have already occurred, they have rarely been guided by CS, creating the risk that the new systems are no more resilient than those they replace or are even maladaptive-for example, increased irrigation in parts of Ethiopia and Kenya when pastoralists have shifted to sedentary agriculture as a response to drying, which leads to depleted rivers or aquifers in the long term (Vermeulen et al. 2018).

The case study from India (Box 1) provides a rare existing example of how CS have informed a transformative shift in agricultural production in India.

Beyond merely improving CS, applying it more effectively to transformative adaptation challenges is crucial. This paper explores how applying properly enhanced CS to adaptation decisions could make case studies like the one above more common, significantly accelerating planning, financing, and implementation of transformative shifts in agricultural systems where and when they are needed. The paper considers what information would be needed and how it should be used. It proposes that when adaptation funding entities, planners, policymakers, and research organizations can access and apply enhanced CS, they will be better able to guide strategic shifts toward long-term resilience in agriculture systems.

\section{Contributions and Challenges of Existing CS for Agricultural Adaptation}

Existing CS, which commonly include weather forecasts, warnings, and agrometeorological advisories, are playing an important role in climate resilience and have generated a substantial and growing body of knowledge about climate change and its impacts on agricultural production. CS have helped farmers to better anticipate and manage extreme weather events like floods, drought, and storms and to take advantage of favorable conditions for planting, harvesting, and other annual milestones. CS for farmers are sometimes combined with non-meteorological data like agricultural production statistics, market conditions, and extension advice to create more comprehensive advisories and guidance.

Expanded funding for CS has driven a significant evolution over the last two decades, leading to improved forecasting capability, better understanding of climate and Earth systems, and growing demand for information to guide climate risk management. Additionally, advancements in communication technology (e.g., mobile phones, apps, and online platforms) have made CS more accessible than ever. This is largely thanks to support from the donor community: Collectively, across four of the largest climate funds' portfolios-the Green Climate Fund, the Adaptation Fund, the Pilot Program on Climate Resilience, and the Least Developed Countries' Fund-climate services feature among the top four focus areas in their project portfolios (WRI 2018).

The process of developing CS involves climatologists, meteorologists, remote sensing specialists, agronomists, hydrologists, communication experts, economists, planners, extension officers, farmers and livestock owners, and other stakeholders-and, increasingly, computer scientists and data experts. Effective CS require sustained engagement between information providers and users to understand user information needs and involve them in the coproduction, delivery, and evaluation of CS (Tall et 


\section{Box 1 | Case Study: Revival of Millet Production in India}

Millet, often regarded in India as a "poor man's food," is a hardy, small-seeded crop that grows well in dry and drought-prone regions and does not completely depend on inputs, such as irrigation and fertilizer. However, in many areas of India, millet has been displaced in recent decades by crops like paddy and maize. In response to current and expected future climate-change impacts, farmers, supported by a range of government and NGO efforts, are reviving millet production in India to help increase the capacity to adapt to climate-change impacts and improve the country's nutritional security, especially as the climate becomes drier (Bose 2018)

Based on interviews conducted with the implementing NGOs, Watershed Support Services and Activities Network (WASSAN) and the DHAN Foundation, programs to revive millet production in India have taken a systemic, three-pronged approach to transforming millet production, governance, and consumption:

1. Shifting back to millet from more recent water-intensive crops like paddy and maize, which will become less viable under projected future climate conditions (Hijoka et al. 2014) in regions that traditionally grew millet.

2. Structural changes to the food security governance system by including millet in large-scale government procurement programs and the public distribution system, as well as incentivizing farmers to grow millet by including the crop under the minimum support prices mechanism.

3. Changing consumption patterns to include millet through awareness programs on nutrition and distribution of recipe books in local languages. The program also supports local processing centers and bakeries to create new millet-based products to increase the demand for millet.

The revival of millet was spurred by analysis of climate data. In selected areas, the Indian Meteorological Department (IMD) has collected climate data over the last 40 years and automatic rainfall station data over the last decade. Data analysis showed that although annual rainfall totals have remained largely the same, there has been a decline in the number of rainy days and delayed onset of the monsoon, resulting in crop failures. These trends are expected to continue: IMD's long-term climate projections point to delays in the onset of monsoon season continuing, as well as further variability in the rainfall patterns (Government of Tamil Nadu 2019). The NASA Earth Exchange Global Daily Downscaled Projections and Coordinated Regional Climate Downscaling Experiment datasets for these regions also project no or a slight variation in cumulative precipitation but an increase in dry spells and extreme precipitation days (PREPData 2018). These climate data, combined with crop models, indicate that rice, wheat, and maize may become less viable as climate change continues while millet is better able to maintain productivity by the 2050s (Gupta et al. 2014; Knox et al. 2012).

Based on long-term climate projections and local weather forecasts, the NGOs and other decision-makers took the following actions (WASSAN 2017):

- Encouraging farmers to plant drought-tolerant, small varieties of millet, such as Finger, Little, Barnyard, and Kodo, in place of cotton, maize, and pigeon pea.

- Encouraging farmers to delay sowing due to later onset of monsoon rains.

- Concentrating efforts to conserve traditional drought- and heat-tolerant varieties of millet by establishing local seed centers to identify and distribute varieties of millet with these characteristics to local farmers.

- $\quad$ Supplying farmers with daily and seasonal weather information to increase production (pest management, protective irrigation, timely fertilizer application) and protect produce stored on farms.

The role of long-term climate projections has been instrumental in determining which areas would benefit from a revival of millet cultivation, as well as in informing different cropping practices. Ongoing discussions between farmers and IMD and proper dissemination of climate information by NGOs, such as the DHAN Foundation and WASSAN, also played a key role in getting farmers to consider long-term climate trends and projections in their decision-making processes. This case study also highlights the potential for reviving traditional knowledge and indigenous practices to support farmers in drying conditions to engage in transformative adaptation.

Source: Namrata Ginoya and Parvathi Preethan. 
al. 2014b). By developing sustained engagement among planners, decision-makers, and climate researchers, better alignment of research agendas and planning process timelines can be achieved. The collaborative process required for effective CS can contribute more broadly to creating equitable, inclusive, and sustainable agricultural policies, investments, programs, and projects.

At the national level, National Meteorological and Hydrological Services (NMHS) are key entities for developing and providing CS. NMHS, by international agreement under the auspices of the World Meteorological Organization (WMO), are the authoritative source of weather, climate, and water information. Linkages among NMHS, government ministries, universities and academia, international CS organizations, boundary or intermediary organizations (those that facilitate access to and use of CS and interaction between CS producers and users), and users should facilitate work on CS coproduction and delivery for agricultural stakeholders (Harvey et al. 2019). Additionally, agricultural universities often play a critical role in climate research and act as hubs for accessing CS. The private sector is also increasingly involved in CS, often through information tailoring and dissemination but also through production and as users (Usher et al. 2018).

While there is a global effort to modernize CS, the lack of CS systems that effectively, iteratively, and over the long term bring together the range of stakeholders to coproduce fit-for-purpose, user-friendly climate products remains a gap. Despite increased investment in recent years, CS systems often lack the strong links and partnerships among CS researchers and providers, ministries of agriculture, intermediaries, social scientists, user communities, and others that are a vital precondition for improving CS generally and, more specifically, to better tailoring it to support transformative adaptation in agriculture. This challenge is related to inadequate policy, legal, and funding frameworks to support CS at national levels. Additionally, NMHS need institutional strengthening to be able to meet the evolving demands for CS in the context of transformative approaches.

While robust evidence of the value of applying CS is limited (in part because CS are just one of many agricultural inputs in complex socioeconomic systems), many argue that CS are a high-value investment (Tall et al. 2018). Applying CS has demonstrably led to increased yields and incomes as well as time saved, more efficient allocation of resources, and improved decision processes (Vaughan et al. 2017). Evidence from 16 studies in sub-Saharan
Africa demonstrates that using CS in agricultural decisionmaking resulted in gains of up to 66 percent in yields or income (Vaughan et al. 2017).

CS have also proved their value through early warning systems critical for agricultural production and food security (IPCC 2019). Early warning systems can help avoid agricultural losses from devastating natural disasters, such as the droughts, floods, and storms that led to US\$96 billion in damaged or lost crop and livestock production in developing countries between 2005 and 2015 (Conforti et al. 2018). In Mongolia, early warning of severe winter storms following a drought period in 2017 led the Food and Agriculture Organization of the United Nations (FAO) to trigger an early action response (e.g., supplemental feed for livestock). An FAO assessment found that for every dollar spent on feed interventions, households had a return of $\$ 7$ in avoided losses and added benefits (FAO 2018). CS for early warning are also being applied to trigger international support to governments ahead of forecasted climate stress and disasters. For example, the World Food Programme's Food Security Climate Resilience Facility initiates action based on climate forecasts to reinforce community resilience before shocks occur. Analysis suggests that this kind of support could reduce emergency response costs by approximately 50 percent (WFP 2016).

Improving CS holds great promise for achieving even greater benefits. The World Bank, for example, estimates that improving CS could increase global agricultural productivity by up to $\$ 30$ billion per year (Anderson et al. 2015). Assessments have found cost-benefit ratios (return on investment) ranging from 1:2 to 1:10 for investments in national meteorological services in Central Asia, Europe, and the United States (Perrels et al. 2013; Rogers \& Tsirkunov 2013). Rodrigues et al. (2016) estimate that national seasonal forecasts adopted by all farmers in Kenya, Malawi, Mozambique, Tanzania, and Zambia would generate regional GDP gains averaging \$113 million per year ( $\$ 3$ per hectare) relative to no access to forecasts.

Despite the substantial improvements that climate services have undergone in recent years and their demonstrated value for agricultural adaptation, CS face many challenges globally that need to be resolved to meet the challenge of intensifying climate-change impacts and address the information needs for transformative adaptation in agriculture. This paper argues that one of the most important challenges for applying transformative adaptation in many developing countries is the state of the 
NMHS, which has generally deteriorated over the past two decades due to underfunding, weak political mandates, and increasingly outdated and deteriorating technologies (Rogers and Tsirkunov 2013; Hansen et al. 2019). Strengthened NMHS will be critical for generating open data, models, and services that promote transformative adaptation, as well as acting as key players in transformative pathways as they will often be the sources of information that policymakers may consult. There is a need to modernize NMHS in more than 100 countries, half of which are in Africa (Rogers and Tsirkunov 2013). Modernization efforts needs to address the following specific institutional and technological challenges:

Limited institutional capacity and lack of robust partnerships and institutional arrangements among stakeholders (from service providers to users) to effectively and equitably facilitate CS that meets growing and changing information needs among diverse users

Under-investment and insufficient attention to users, which leads to inadequate systems, poorly designed products, and limited accessibility

- Limited observation infrastructure and the associated data scarcity and lack of reliable historic observations in developing countries

- Limited data sharing among international, regional, national, and other entities

Unsupportive policy and legal environments for CS

- Limited model downscaling

- Lack of access to robust data storage and computing infrastructure to run computer heavy models

Lack of reliable information delivery systems, including through information and communication technologies (ICT)

Wide disparity in scientific capacity and the availability of CS, including between developed and developing countries (Singh et al. 2018; Singh, Dorward, \& Osbahr 2016; Tall et al. 2014a; Georgeson et al. 2017; Harvey et al. 2019; Hansen et al. 2019)

\section{ENHANCING AND APPLYING CS TO ADVANCE TRANSFORIMATIVE ADAPTATION}

Much of the substantial and growing body of knowledge about climate change and its impacts on agricultural production points to the need for transformative adaptation. Climate-change impacts to food production systems are expected to become increasingly severe after 2030 and more so after 2050 as trends affect yields, interannual variability, and the amount and location of arable land (FAO 2016). Temperature and precipitation trends, climate extremes, and sea level rise are already suppressing yields for key crops around the world with the largest impacts on wheat and maize and in tropical regions (Hoegh-Guldberg et al. 2018). Globally, warming of $2^{\circ} \mathrm{C}$ is expected to cause a loss of 7-10 percent of rangeland livestock with significant associated economic losses and negative impacts for communities and regions in much of the world (Hoegh-Guldberg et al. 2018). More specifically, in sub-Saharan Africa, for example, climate projections show that critical thresholds for some agricultural systems may be crossed as soon as 2025, to the detriment of livelihoods and food security (Rippke et al. 2016).

Enhancing CS beyond the current focus on short-term planning to advance transformative adaptation will require developing CS with information to better support medium- to long-term planning in agriculture. Investments in long-term, systemic change need to be based on salient climate information with the aim of supporting those most vulnerable to climate-induced shifts to agricultural systems. Enhanced CS should be integrated into decision-making at the global, regional, national, and subnational levels and, ultimately, applied at the farm level. Good scientific information, understanding capacity needs at different levels, a robust monitoring and evaluation system, and CS coproduction are as key to achieving transformative adaptation goals as they are for meeting adaptation objectives more generally.

CS that consider transformative approaches to adaptation could build off the successes of early-warning systems. Analysis of the frequency and magnitude of extreme events may indicate that certain regions are getting close to tipping points at which they will essentially become different ecozones-for example, shifting from semi-arid to arid as droughts increase in frequency and duration. Early-warning systems could be designed to signal such system changes by including information to indicate that specific thresholds are being reached-for example, 
Enhanced CS for transformative adaptation incorporates the following elements

New types of information to determine transformation hotspots (e.g., crop or livestock climate suitability thresholds, scenarios of increased frequency of

extreme events, market projections)

Expanded time horizons (e.g., longer-term scenarios beyond 10 years; short-, medium- and long-term adaptation options)

Bundled information that enables transformative planning and decision-making (e.g., land tenure, available insurance schemes, market niches, and

linkage opportunities for novel crops, information about analog systems)

Tailored, yet complementary, services for different transformative pathway actors (e.g., long-term scenarios for policymakers, market niches for public

and private funders, crop and input value chains for development planners)

multi-year trends of declining precipitation, higher temperatures, and lower yields of existing varieties of crops and livestock. This would warn adaptation planners and funders that they should begin channeling funding toward adaptation interventions more suitable for the new climatic conditions and encourage transformative change, rather than continuing to invest in maintaining agricultural systems that are losing viability in particular regions. Services like the Famine Early Warning System Network that combine agroclimatic monitoring with scenario development, typically over annual time frames, could be enhanced by providing increased focus on longer-term outlooks regarding agricultural system viability.

An increased focus on CS for longer time frames should come in addition to, not at the expense of, the daily to seasonal forecasting that is critical to existing agricultural systems and sequencing short- and medium-term actions along transformative adaptation pathways. In fact, much investment in CS systems (e.g., infrastructure, research, capacity, and information sharing) will improve climate information and CS products across multiple time frames. Relevant CS infrastructure and capacity investments that could improve both short- and longer-term CS include

rehabilitating and reequipping observation and monitoring infrastructure;

updating information technology software and hardware;

- increasing high-performance and cloud computing, data storage, and digital data connectivity; and

- improving information product-development and -sharing mechanisms.
Without such improvements, decision-makers, policymakers, funders, and practitioners may continue to invest in agriculture systems that will not withstand longer-term climate impacts instead of investing in new systems where and when they are needed. This could cause agricultural systems to be transformed into unsustainable alternatives or contribute to maladaptation that actually increases vulnerability-for example, by investing scarce resources in irrigation infrastructure to deliver water supplies that projections indicate are likely to dry up in the coming decades, rather than shifting to crops and livestock more suitable for hotter, drier conditions.

CS could also be enhanced to overcome another shortcoming of current information systems: Although they are informative regarding which types of agricultural production are expected to become less productive, they rarely provide decision-makers with essential information on which types of crops and livestock might become more productive in particular locations as the climate changes. (See Box 3 for a promising exception.) This information could be included in CS for transformative adaptation, along with other types of information that are currently rarely included, such as decadal and longer-term climate projections, agro-climatology tools that combine climate and crop parameters, climate-risk and vulnerability analyses, climate projections, climate scenarios, and more.

Additional information gaps further challenge the application of CS in transformative adaptation. There is a need to improve and downscale climate research and crop and livestock models that can best determine where and when agricultural systems will exceed thresholds, the options for alternative systems, and incremental measures along transformative pathways that would pave the way for transitions from current agricultural systems to those 
better suited for anticipated conditions. For crop models, a focus on both cash and staple crops is critical, and expanding coverage to include more traditional nonmarket crops would be very useful. A better understanding of the resource implications and long-term trade-offs of particular transformative pathways across sectors is also necessary to inform decisions about alternative systems under various climate scenarios.

Further, as agricultural systems shift, so does land value and competition for productive land, which highlights the need to consider land-tenure systems and conflicts in transformative adaptation approaches. Land-tenure systems influence people's and communities' vulnerability and adaptive capacity (IPCC 2019). As the IPCC Special Report Climate Change and Land notes with medium confidence, "Insecure land tenure affects the ability of people, communities and organisations to make changes to land that can advance adaptation and mitigation" (IPCC 2019, p. 31). CS bundled with land-tenure information systems (e.g., WRI LandMark) could help inform transformative adaptation approaches at the program level that work to secure land rights and anticipate and avoid land conflicts among various stakeholders.

The level of investment in CS required to adequately monitor weather and climate and inform transformative adaptation for agriculture has not yet been determined. Although no explicit evidence exists to quantify the costs and benefits of developing and deploying CS to inform transformative adaptation, such investments have the potential to pay off as climate impacts intensify and the limits to incremental adaptation are reached in more and more existing agricultural systems.

\section{Applying CS to Build Transformative Pathways}

Shifting agricultural systems toward climate resilience over the longer term will require decision-makers to employ transformative pathways - that is, coordinated sequences of short- and medium-term actions or projects that can be phased in gradually to shift agricultural production systems to become more resilient to longerterm projected future climate impacts, while allowing sufficient flexibility to respond to new information (Carter et al. 2018). As adaptation entities develop transformative pathways, CS tailored for this purpose could inform

where shifts in climate variables are expected to reach tipping points and exceed the thresholds of agricultural systems as they are currently managed (e.g., where temperature thresholds for maize or coffee will be exceeded, or where rangeland resources become insufficient to support cattle), and thus transformative adaptation is likely to be needed;

which crops, livestock species, methodologies, and technologies or systems are most likely to be viable (and which are not) in future climate conditions in different locations;

when these thresholds or tipping points may be crossed, and shifts will need to occur; and

what incremental options (e.g., policies and practices) can be sequenced to gradually phase in longer-term transformation in farming and pastoral communities and along their agricultural value chains.

Work to improve the accuracy of climate modeling is ongoing, but even so, uncertainties will inevitably remain, such as those associated with the rate of global socioeconomic development and overall global GHG emissions, including the effects of unexpected events like the current COVID-19 pandemic and other types of shocks. In addition, agriculture viability thresholds are difficult to model accurately over longer time frames given the range of relevant factors, including soil moisture, number of dry days in climate-sensitive growth phases, water availability, pests, and more. To best apply enhanced CS to long-term planning, decision-makers will need appropriate guidance, including approaches like robust decision-making, an iterative decision framework designed to highlight vulnerabilities and trade-offs in situations of uncertainty (Bhave et al. 2016). Further, transformative adaptation pathways themselves are necessarily designed to allow for adjustments in response to new information.

Developing transformative pathways requires stakeholder engagement in testing and assessment of potential activities, as well as innovative approaches to scaling. Stakeholder engagement also enables local environmental and socioeconomic conditions critical to the efficacy of adaptation efforts to be taken into account (IPCC 2019). Using the areas and agricultural systems projected to require transformation by 2050 as a starting point, contextspecific transformative pathways could be guided by CS to inform appropriate policies, systems, and practices. This would require

engaging the key decision-makers (CS providers, adaptation planners, support entities and implementers, intermediaries, and farmers) in sharing information on current challenges and the apparent effectiveness of responses that are being tested; 
considering and selecting possible transformative pathways based on climate projections, crop and livestock monitoring and models, and the extent to which existing interventions are likely to be effective;

- identifying decision-maker information needs for the target transformative pathways and preferred communication channels (including using appropriate technologies to increase reach);

collaboration among NMHS, other CS providers, and key decision-makers in coproduction of the needed $\mathrm{CS}$; and

- ensuring that CS are accessible and combined with appropriate application support for the range of activities along the pathway.

Rippke et al. (2016) offer an example of how climate research could be used to inform components of a transformative pathway (Box 3) and a series of steps for developing and enacting it. In this model, movement toward transformative adaptation is informed by short-, medium-, and long-term climate information as well as information on market opportunities, risk-transfer options, and more. Combining short-, medium-, and long-term climate information allows for decision-making across spatial and temporal scales that considers evolving climate risks and adaptation opportunities. Well-informed and low-risk incremental actions build in flexibility for adjusting adaptation pathways and provide the foundation for long-term transformative adaptation. These incremental actions along an adaptation pathway and built-in flexibility are key for managing the uncertainties and limitations inherent in global climate models (Nissan et al. 2019).

\section{Data Needs and Their Use for Transformative Adaptation}

Transformative adaptation requires decision-makers to integrate long-term climate projections (targeted toward mid-century) into adaptation planning processes, but few decision-makers adequately incorporate such projections and their implications into planning for agricultural systems. Singh et al. (2018) argue that the gap in use of CS for longer-term decisions is due to the lack of fit-forpurpose information and the challenge of putting decadal and multi-decadal projections to use given issues of uncertainty, risk aversion, and the inability to justify investment based on projected risk. This twofold challenge-improving the availability of fit-for-purpose information and the capacity and willingness of decision-makers to use itrequires work to both enhance CS and improve decisionmaking processes.

\section{Box 3 | Utilizing CS to Inform Transformative Pathways}

Combining climate and crop models, research in sub-Sahara Africa assessed options for transformation when and where production of key crops will likely become nonviable. Up to 30 percent of areas growing maize and bananas and up to 60 percent of beanproducing areas are projected to cross productivity thresholds by 2100 and become nonviable in some areas as soon as 2025 (Rippke et al. 2016). Conditions exceeding system thresholds are particularly prominent across crop types in the southern Sahel by the 2050 s and in bean growing areas of East Africa, mainly after the 2050s (Rippke et al. 2016). In Uganda and Tanzania, about 10 percent of suitable bean-producing areas are likely to become nonviable by the 2050s and 30 percent by the 2090s (Rippke et al. 2016). In contrast, root crops (yams, cassava) and drought-resistant cereals (millet, sorghum) are projected to be less affected by climate trends, with less than 15 percent of the currently suitable area crossing a productivity threshold by the 2090s (Rippke et al. 2016).

Based on future climate suitability, Rippke's research suggests that maize farmers may shift to more drought-tolerant cereals like millet and sorghum, which are viable substitutes in all but a few locations, although they may experience some yield reductions. In some areas of the southern Sahel and southern and eastern Africa, however, more significant shifts from crop to livestock systems will be likely (Rippke et al. 2016). The research proposes a framework for implementing transformative pathways in crop systems that involves three overlapping phases: an incremental adaptation phase that focuses on improvements to existing crops and management practices; a preparatory phase that establishes enabling environments at multiple levels to support transformational change; and a transformation phase in which farmers substitute crops or explore alternative livelihood strategies.

This research demonstrates that CS are critical for informing options for alternative systems suited to various climate scenarios, the optimal incremental adaptation measures along the way, and monitoring the threshold parameters that trigger the shift to the next phase (tipping points). The research identifies the following thresholds for movement into the subsequent phase:

Business-as-usual to incremental adaptation phase-climate variables make 5 years out of 20 unviable but further increased nonviability is not projected until late this century

Incremental adaptation to preparatory phase- climate variables make 5 years out of 20 unviable and further increased nonviability for 10 of 20 years is projected in the next two decades

Preparatory phase to transformational change-climate variables make 10 years out of 20 unviable (Rippke et al. 2016) 
Lack of fit-for-purpose information: Although work remains in identifying information needs for transformation and creating applicable models and products, researchers are increasingly producing fit-for-purpose information that can inform transformative pathways. The integration of longer-term climate projections with crop models and other relevant information sources (Box 4) is a key example of this type of fit-for-purpose information.

Additionally, enhanced CS could accelerate the spread of potentially transformative agricultural technologies and practices if bundled with additional types of information. For example, precision agriculture and in-season field and water management strategies, which are not yet widely used in the developing world, use CS to track and time field-level responses to water stress, heat stress, excessive water, wind damage, and more. Weather index insurance and satellite-derived crop insurance also use CS to develop and monitor the index thresholds that trigger payouts, such as cumulative rainfall.

Inability to justify investments: Although CS relevant for transformation are on the rise, limited integration of these climate services and comprehensive models into planning remains an obstacle. Reasons for the limited integration of long-term climate information into planning are multiple and complex. Data users tend to be oriented toward climate information designed for short-term decisions with immediate results. Part of this orientation is related to the psychological distance of longer-term climate-change impacts, given the uncertainty of when, where, and how an impact might manifest itself. Additionally, given the level of uncertainty intrinsic in climate projections, acting on these projections in complex contexts "pits an unsure gain against an unsure loss," creating difficult decisions regarding changing behavior (Jones et al. 2014). Although climate-change impacts are increasingly experienced at local levels, impacts are ever-evolving, and many have yet to manifest themselves. Experience is often a stronger driver of risk perception and behavior change than analytical thinking. When a risk has not yet been experienced, people are not likely to have a strong emotional response and will delay decisions and behavior change to address the risk (Slovic et al. 2002).

Additionally, decision-makers often face a lack of guidance and limited capacity to consider long-term climate risks, design transformative pathways, and apply climate information to sequenced adaptation actions. Increasing research on and investment in understanding government and other planners' information needs for transformative
Box 4 | Climate Projections and Crop Models

Climate information over multi-decadal time frames is critical

to helping inform where and when climate impacts threaten

agriculture to such an extent that transformative approaches are

required. Climate projections rely on climate (i.e., general circulation

models) and Earth systems (ecological, hydrological, and economic,

etc.) models to determine a range of likely future conditions,

depending on the level of ongoing GHG emissions. Researchers are

developing increasingly comprehensive models to apply CS in crop

production over a range of time frames. FAO's Modeling System for

Agricultural Impacts of Climate Change (MOSAICC) is a powerful

tool that integrates climate, crop, hydrology, forest, and economic

models for national-level climate-change impact assessments.

National assessments examine the threats and opportunities

that climate change presents for agriculture to inform adaptation

efforts (FA0 2015). FA0 has worked with interdisciplinary technical

working groups in eight countries to use MOSAICC for evidence-

based national adaptation plans, projects, and programs. Less

complex models, such as FAO's EcoCrop, are also able to produce

spatial suitability projections of more than 2,300 species. Additional

climate-crop models and how the adaptation community might

leverage them for transformation are described in Appendix B.

adaptation in agriculture can facilitate development of models, tools, guidance, capacity building, and decision support for applying enhanced CS. Approaches such as decision support matrices, climate narratives, or scenario methodologies can also support decision-makers in using enhanced CS to identify transformative adaptation pathways appropriate for the range of potential future climate conditions (Bhave et al. 2016; Dessai et al. 2018).

Examples of CS informing long-term decision-making at national and subnational scales are limited but do exist, often in the private sector. In a growing number of cases, agricultural businesses are using climate information as a deciding factor for undertaking transformative approaches. In Australia, for example, CS were used to persuade producers to incorporate new crop types and relocate production (vineyards and peanuts) to areas where growing conditions are projected to be better in the long term (IPCC 2014). With recognition that climate change could halve the area suitable for coffee production by 2050 , more than 40 private-sector, conservation, and research institutions have formed the Sustainable Coffee Challenge. The group monitors how areas suitable for coffee production are changing and are expected to change over time to inform opportunities for sustainable produc- 
tion. The group has also committed to improving farmer access to inputs, finance, and climate-change information (Sustainable Coffee Challenge 2019).

Although the use of CS for transformative adaptation has great potential to protect food security and incomes, climate information may also be used to alter agricultural investments that could leave producers behind. The public and private sector may use CS for climate-risk mitigation that could result in shifting agricultural investments to new geographies and products. Cocoa investments, for example, may move from West Africa to Central America, while additional investments in climate-sensitive products could be abandoned in favor of more resilient products. Anticipating these shifting investments will be key to supporting affected populations in adapting to both climate and market impacts.

While examples such as these make it clear that CS that combine longer-term climate projections with crop models can inform transformative changes, there are relatively few examples of such systems being used by developing countries for agricultural planning. In addition to better guidance and capacity building, this may point to a need to more thoroughly explore opportunities to leverage private-sector engagement in CS to support transformative outcomes, which could then be shared with developingcountry governments. Carefully designed public-private partnerships can help address CS challenges, including underinvestment and poor infrastructure, services, and capacity, as well as increase the range of services available to users. The private sector has the potential to bring new revenue streams and financial sustainability to CS systems, as well as networks and expertise. Opportunities for engagement include public-private partnerships with private-sector actors such as CS businesses, agribusinesses, food and beverage companies, and input suppliers. Some food and beverage companies, for example, are tailoring CS to increase the long-term resilience of their supply chains (Sloan et al. 2018). There may be opportunities to make some of this information public in a precompetitive space and increase sustainability more broadly without compromising profits.

One area where private-sector engagement in CS has yielded widespread benefits is in working to build robust historical and contiguous climate datasets to support long-term climate risk assessments. For example, the company aWhere delivers agricultural information and "weather intelligence for a changing climate" to agriculture users around the globe. aWhere products support daily decision-making as well as monitoring of long-term shifts in agricultural production zones (aWhere, Inc. 2020). IBM has developed a climate-risk and resilience services platform and approach to provide businesses with climate-related risk management, scenario analyses, and solutions to define, prioritize, and address climate risk and associated investments and value-chain initiatives. IBM's Climanomics web-based software allows users to identify climate risks and trends and integrate climate risk into operational decision-making (TCS 2020). Additionally, companies like Climacell are increasingly using artificial intelligence-powered software to offer planning support based on historic, real-time, and future weather. Driving further long-term climate risk assessment in the private sector, the G20 Task Force on Climate-Related Financial Disclosures has recommended that companies and other organizations report on climate-change risk in order to support informed, efficient capital allocation decisions. These private-sector initiatives have the potential to be valuable partners in supporting the information infrastructure required for transformative adaptation in agriculture.

In addition to these agricultural business examples, some agricultural research and support organizations are beginning to use CS to develop transformative adaptation programming. In Nicaragua, for example, the International Fund for Agricultural Development (IFAD) Adaptation for Smallholder Agriculture Program has supported widespread agricultural diversification away from coffee in areas expected to exceed coffee production thresholds in coming decades (IFAD 2014). In Ethiopia, IFAD has supported substantial expansion of small-scale irrigation in response to projected increases in drought occurrence and intensity. The Consultative Group on International Agriculture Research (CGIAR) has also developed a full program approach for applying CS to transformative adaptation in agriculture (Box 5).

Ensuring equitable access to information production and use is critical to inclusive transformative adaptation that avoids consolidation of wealth and power, which could otherwise further harm marginalized groups and contribute to the "climate apartheid" that a recent UN report sees emerging (United Nations Human Rights Council 2019). Supporting equitable access means investment in enhanced CS in developing countries, with particular attention to making CS accessible to and applicable for marginalized populations. Attention to issues of equity is also critical when engaging the private sector. Broad agreement is needed on which CS components and 


\section{Box 5 | Leveraging CS to Identify Analogue Sites for Transformative Approaches}

In response to the need for transformative adaptation, some

agricultural research and support organizations are using CS to

design new approaches. The climate analogues approach brings

climate projections to life by identifying real-world examples in one

area that demonstrate the climate future of another area. CGIAR's

Climate Change, Agriculture, and Food Security program (CCAFS)

"farms of the future" approach uses climate scenarios to identify

areas likely to exceed system thresholds, and analogue areas with

climates similar to the target areas' projected climate (CCAFS 2016).

CCAFS then organizes exposure visits for target-area community

leaders to explore and learn from the experiences and agricultural

approaches and practices in the analogue area. In an example

from Senegal, the "farms of the future" approach has led women

from the increasingly drought-prone Daga-Birame village to protect

the area's baobabs, cultivate new ones, and adopt other drought-

resistant, fruit-bearing trees, such as jujube, tamarind, guava, and

soursop. Through increasingly producing, processing, consuming,

and marketing drought-resilient tree crops, the women are in the

process of transforming the agricultural system (CCAFS 2016.

products should be provided as a public service versus cost-recoverable services. But even with this, the private sector generally has greater capacity to use information from publicly available CS, as well as more ability to invest in exclusive CS, both of which could increase the information gap between wealthy and marginalized agricultural stakeholders. For this reason, public-private partnerships and associated policies and regulations to ensure equitable access need to be tested and proved before wider implementation.

\section{Integrating Enhanced CS into Adaptation and Development Plans}

Despite the challenges, countries are demonstrating their interest in better integrating climate change across sectors in plans to update NDCs in 2020. There are a limited number of examples of national-level plans that incorporate more transformative approaches to adaptation that are based on long-term climate projections and increasingly comprehensive climate and Earth systems models (Graham et al. 2015). In developing NAPs, for example, a few countries are using tools that incorporate climate projections in meaningful ways, such as the World Food Program Consolidated Livelihood Exercise for Analyzing Resilience tool used in Sri Lanka. In Southeast Asia, stake- holders from Cambodia, Vietnam, and Laos have come together to produce development scenarios based on projected socioeconomic developments and climate impacts (CCAFS 2016). These scenarios describe a range of future conditions to help policymakers explore adaptation policy and investment options applicable across climate and development scenarios. The Cambodian Ministry of Agriculture, Forestry and Fisheries applied the scenarios in developing Cambodia's Climate Change Priorities Action Plan for Agriculture, Forestry and Fisheries 2016-2020. Similar scenario planning efforts for policy are being used in Bangladesh, Burkina Faso, Colombia, Costa Rica, Ghana, Honduras, Tanzania, and Uganda (CCAFS 2016). Box 6 provides another example of this kind of analysis, providing critical information on the viability of coffee in Costa Rica.

Climate services tailored to guide and inform major agricultural shifts are needed to better meet the needs of agricultural adaptation funding entities, planners, policymakers, and research organizations. This would require that adaptation planners, government entities, and farmers identify their specific information needs and coproduce information systems in collaboration with others. NMHS, supported by regional and global climate centers, for example, might increase focus on creating long-term projections; while line ministries, agricultural research and support organizations like FAO and the International Food Policy Research Institute (IFPRI), and academia could improve understanding of the implications for system thresholds in key agricultural systems. These information providers could then work with agricultural adaptation planners to identify incremental steps toward transformed agricultural systems and request financial support from funding entities. Similar processes could be used to better integrate the use of enhanced CS into plans for achieving the SDGs, as well as other development planning processes.

One model for this kind of policy-linked research and development of climate services is the European Union's (EU) Horizon 2020 climate services program. Horizon 2020 is the biggest EU research and innovation program ever, with nearly $€ 80$ billion of funding available over seven years (2014 through 2020). Approximately 30 percent of this funding is for climate-related research and innovation, including on climate services. Specific research priorities for Horizon 2020 have been developed through large-scale multistakeholder processes and emphasize user applications and engagement (European Commission n.d.). These priorities have included research 
In 2018, the Ministries of Agriculture and Environment of Costa Rica collaborated with the World Resources Institute (WRI) and other stakeholders to strengthen the coffee sector's climate resilience through transformative adaptation planning. Visualizing data through PREPdata, an open-access climate data tool, was an essential part of engaging with ministry officials and coffee farmers to illustrate important risks, trade-offs and vulnerable areas. Coproduction of regional dashboards allowed for more context-specific evaluation of current and future climate conditions-for example, changes in precipitation and temperature-with other relevant physical and socioeconomic data. The ability to show coffee suitability under various carbon-mitigation pathways allowed stakeholders to assess different adaptation options in the short, medium, and long term and to begin to identify which measures should be employed, when, and by whom (Tye and Grinspan 2020). This type of climate data platform and analysis provides a strong foundation for planning, financing, and implementing transformative adaptation to improve farmer resilience. Similar data and dashboards can be housed and developed in PREPdata for other regions.

Figure B6-1. Projected Changes in Costa Rica's Coffee Suitability by 2050 under a Moderate Emissions Scenario (RCP 4.5)

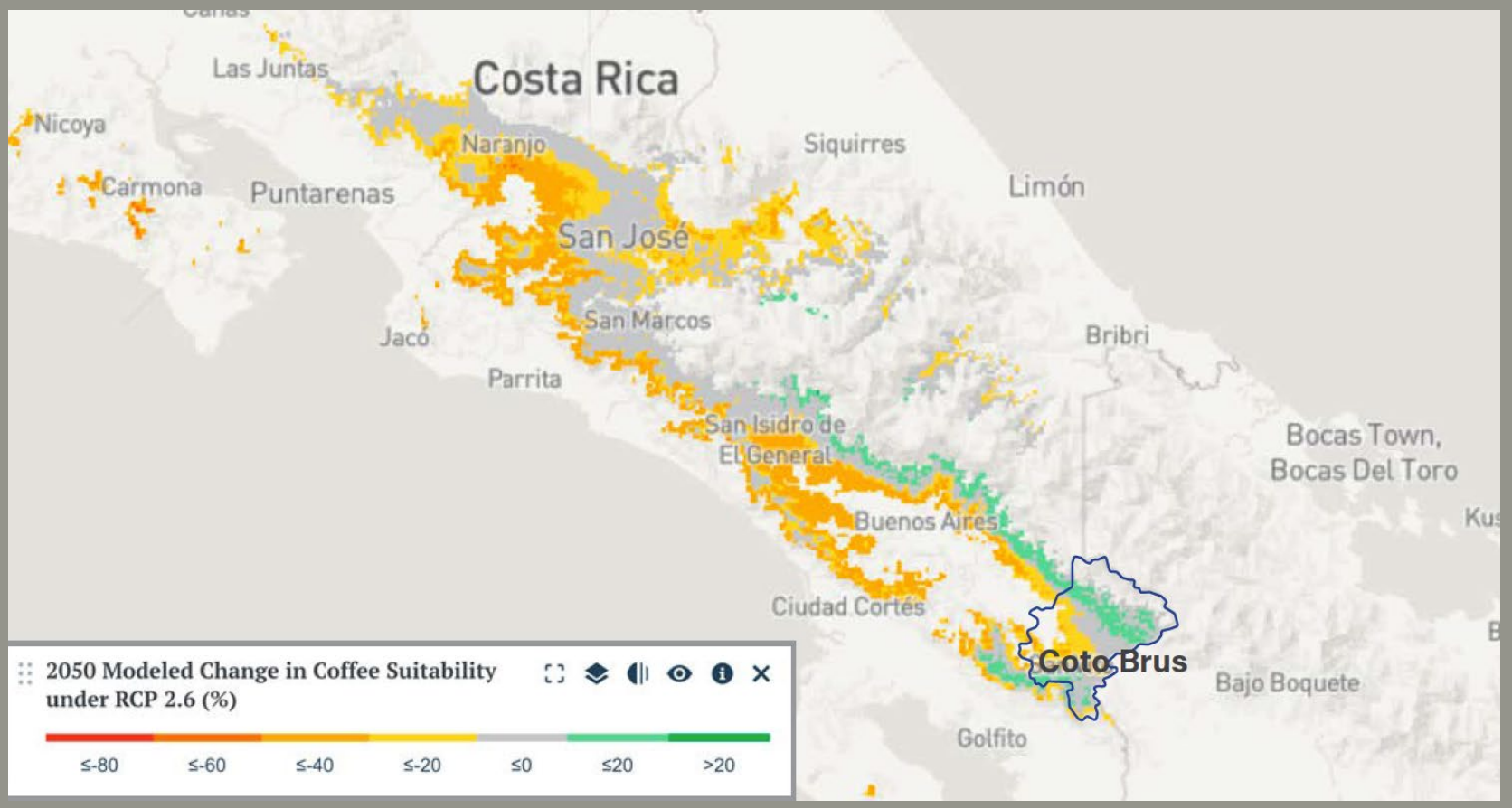

Note: Yellow and orange areas indicate a decrease in suitable growing areas for coffee.

on the impacts of more significant changes in the climate at 4- and 6-degree thresholds through the High-End cLimate Impacts and eXtremes (HELIX) project. Integrating the climate information-service requirements of transformative adaptation into this kind of program would accelerate collaboration, innovation, and progress toward more science-based planning for transformative adaptation in agriculture

For examples like these to become the rule rather than the exception, CS tailored in the ways described must be made more accessible and easier to use, which will require greater investments and action by research organizations, governments, adaptation funding entities, and the private sector. Strengthening boundary organizations that can translate climate science into sector-specific usable CS is critical. Such tailored CS could also be used by adaptation funding entities to inform their funding allocation processes, for example, by linking their investments to achievement of specific milestones along transformative pathways. Policymakers and support organizations could provide policy and technical support for reaching the same goals.

More broadly, development planners need long-term information about food systems, water supply, urban 


\section{Box 7 | Gender, Equity, and Applying CS to Transformative Adaptation}

Inequitable participation in the creation and application of CS for transformative pathways could reinforce existing inequalities and inefficiencies in food production over the long term and limit the positive potential of transformative adaptation. Significant shifts in agricultural landscapes can risk the consolidation of power and wealth as those with resources, including information, may be more capable of transforming while the poor and most vulnerable may be left behind. Alternatively, engaging women and marginalized groups in coproducing and applying CS can lead to transformative adaptation in agriculture that empowers women and marginalized groups through appropriately tailored information products and services.

The differing roles and control of resources among women and marginalized groups in agriculture can make their climate information needs and preferred communication channels distinct from dominant groups. While efforts toward gender inclusion in CS coproduction continue, women typically have less access to CS and particularly to information that is specific to their needs (WM0 2015). Women farmers' access to particular communication channels can be restricted by gender and socio-cultural differences in social norms, literacy rates, and household responsibilities, as well as male bias in extension services (Gumucio et al. 2018).

Beyond information access, women also typically have inequitable access to the inputs (e.g., new varietals, land, fertilizer, etc.) that would be required to act on the CS advisories and recommendations (Doss and Morris 2008). Inequitable access to inputs and inequitable dynamics in decision-making around land use and risk-taking may restrict the capacity for women to engage in transformative pathways in agricultural production (Michalscheck et al. 2020; Bullock and Tegbaru 2019).

Actions to advance gender inclusion in CS will have lasting, longterm effects when stakeholders apply CS to designing equitable transformative adaptation pathways. Evidence suggests that to move toward equality, CS initiatives need to actively target women and marginalized groups to identify their information needs, priorities, and preferred communication channels; recognize when gender, age, or social status adversely affect the effective participation of some community members in communications processes and pursuit of alternative processes; and seek to introduce CS in a manner that decreases women's labor and time investment in agricultural and household tasks (Huyer et al., 2017; Tall et al. 2014a). plans, supply chains, and more to identify trade-offs in resource allocation among sectors. Bringing this information together for decision-making at national and regional levels may require new coordination mechanisms, modeling platforms, and open data. Better using enhanced CS to advance transformative adaptation would require cross-ministerial and multisectoral coordination around the benefits, challenges, and implicit trade-offs among various transformative adaptation options, including consideration of resource requirements across agricultural, urban, and energy sectors. Global Framework on Climate Services-supported National Frameworks for Climate Services, which are mechanisms for coordinating, facilitating, and strengthening collaboration among national institutions, have a key role to play in this effort with their mandate to improve coproduction, tailoring, delivery, and use of science-based climate predictions and services. Mainstreaming climate information and adaptation, however, has proved difficult to implement to date due to a range of challenges summarized in Mogelgaard et al. (2018), which include a lack of adequate policy frameworks, leadership, coordination mechanisms, information and tools, and supportive financial processes.

\section{RECOMMENDATIONS}

Enhancing and applying CS to better inform transformative adaptation requires improving it in specific ways; better integrating the data it provides into policy, planning, and practice at regional, national, and subsnational scales; and making better use of it to guide financing adaptation investments. Recommendations for research, policy, and investment priorities to facilitate this follow.

\section{Research Priorities}

\section{CS designed to support identification of where and when climate change will push existing agri- cultural systems beyond thresholds, as well as options for new systems, are needed to inform and guide longer-term shifts; that is, transfor- mative adaptation. Research on projected crops and} livestock productivity is advancing, but additional evidence is needed, including on which crops and livestock will become more viable in specific locations. Estimates of the timing of such system shifts, especially at the decadal and longer-term timescales, will require ongoing adjustment based on global emissions and climate scenarios and should be informed by an understanding of local social, ecological, and economic contexts. More robust data on 
the effectiveness of various incremental and transformative measures are needed to evaluate whether they will be sufficient to build long-term system resilience. Building this evidence base will require stronger partnerships among international, national, and local research institutions to accelerate applied research on the impacts of climate change on agriculture and food systems.

CS to support transformative adaptation should enhance understanding of long-term trade-offs of transformative pathways across sectors and climate scenarios. Transformative adaptation shifts to new agricultural systems may be associated with changes in land, water, and input requirements (e.g., labor, seeds, fertilizer, etc.) that have impacts beyond agriculture. At the same time, climate-change impacts may be changing resource and input availability and/or quality. CS that enable better visualization of multiple, sometimes competing challenges are needed to better understand the resource requirements and market viability of alternative systems, resource availability (e.g., water, suitable land) under future climate scenarios and the associated tradeoffs (e.g., social, economic, and environmental) beyond the agricultural sector.

Economic analyses of the potential for CS to contribute to transformative approaches would improve CS design and provide much-needed justification for governments and funders to increase investments in such information systems. Although no explicit evidence exists to quantify the costs and benefits of developing and deploying CS to inform transformative adaptation, such investments have strong potential to pay off as climate-change impacts intensify and the limits to incremental adaptation are reached in more and more existing agricultural systems. This research should consider the value of integrating other types of information into CS on topics such as markets; health and nutrition; key resources like water; and finance, savings, and insurance options.

Better identification of decision-makers' information needs for transformative adaptation in agriculture and the associated CS interventions is needed to design CS products better tailored to inform transformative adaptation. Open questions include, for example, which types of coproduction processes are most efficient and effective in identifying equitable transformative adaptation options, what tailored CS products decision-makers need for mapping out transformative pathways, and what types of climate and non-climate systems modeling should be prioritized for improvement to promote sustainable shifts. Consultative research can illuminate the challenges and opportunities for CS to support transformative adaptation decisionmaking in complex socioeconomic contexts and across a range of future climate scenarios.

\section{Policy Priorities}

Key actors must advocate for developing and applying CS for transformative adaptation in the most at-risk agricultural systems to avoid substantial losses in agricultural livelihoods and productivity. Policies and processes are needed to bring stakeholders together to envision, develop, and share the CS needed to inform transformative pathways with sequenced actions and built-in flexibility. Capacity must be strengthened to enable users to fully participate in the coproduction and application of CS. As a key CS provider, NMHS, in particular, need support to make institutional shifts from identifying as data collection entities to instead considering themselves public-service providers that incorporate much greater stakeholder engagement. NMHS also need strengthened capacity to work with climate scientists in the modeling, forecasting, and climate product delivery (including information and communications technologies) that support production and integration of long-term information.

Given the knowledge-intensive nature of applying CS, particularly to transformative approaches, policymakers and funding entities need to encourage its application by a range of users. Across scales, decision-makers need stronger guidance and capacity building to encourage them to consider long-term climate risks, design transformative pathways that will often need to integrate across multiple sectors, and apply climate information to sequenced adaptation actions. Adaptation planners, policymakers, funders, and other stakeholders need guidance that makes clear the need to consider longterm climate risks, how to identify appropriate adaptation actions along transformative pathways, and how to translate technical climate and agro-climate analysis into planning decisions. This CS application support will be different from what has thus far been offered to stakeholders and requires a comprehensive approach to increase the capacity to support users. This could take the form of training, courses, exchange visits, peer-to-peer learning, regional events leveraging the WMO regional climate centers, and other learning strategies. 


\section{Investment Priorities}

Governments and adaptation funders need to coordinate and sustain investments in expanded capacity and infrastructure for CS components critical to transformative adaptation, including observations and monitoring; research, modeling, and prediction; coproduction processes and user interface platforms; and technical capacity building. This will require infrastructure investments, including rehabilitating and reequipping observation and monitoring infrastructure, updating information technology software and hardware, and creating automated information and communications technology services.

High-performance and cloud computing and data-storage and digital data connectivity require additional investment. Such investments, along with the associated technical capacity building, will aid in transformative adaptation planning and implementation and, in return, will help to inform investors of where, when, and how to invest in system shifts, including how to phase and compound project outcomes.

Investments in national and regional coordination are key for developing and applying the high-quality, tailored climate services needed to inform transformative pathways for agriculture. Engagement and coordination with entities working at national and regional levels can facilitate collaborative production and application of CS tailored to the local contexts, opportunities, and barriers that influence the success of transformative approaches. Entities such as WMO's National and Regional Frameworks for Climate Services, the Global Framework on Climate Services, and CGIAR's CCAFS program are potential partners in the effort to effectively develop and implement transformative pathways for agriculture.

Exploration of opportunities to leverage privatesector investments in CS could support transformative outcomes and financially sustainable CS systems. Carefully designed public-private partnerships could help to address underinvestment or redundant investment in infrastructure, services, and capacity and increase the range of services available to users. Privatesector initiatives are increasingly using sophisticated climate-risk assessments to inform long-term decisionmaking. Engagement with these initiatives could result in information sharing relevant for the government, development, and farmer communities that are integral to private-sector production. Attention to issues of equity and greater agreement on what CS components and products should be provided as a public service, versus as cost-recoverable services, are key to inclusive transformative adaptation that avoids further consolidating wealth and power. Additionally, engagement with agribusinesses will be important to understand when they are looking to shift agricultural products sourcing from one region to another in order to anticipate and mitigate negative local economic impact. 


\section{PLATFORM OR MODEL DEVELOPER}

Modeling System for Agricultural Impacts of Climate Change (MOSAICC)

Global Agro-Ecological Zones (GAEZ) Global Agro-Ecological Zoning (GAEZ)

IMPACT 3 IFPRI

Decision Support

System for Agrotechnology

Transfer (DSSAT)

\section{CGIAR Platform for Big}

Data in Agriculture

Water Requirement

Satisfaction Index

(WRSI) and Geo-spatial

WRSI

PREPdata

EcoCrop

FAO

FAO and IIASA

DSSAT

CGIAR

FAO Resilience and Preparedness
DESCRIPTION

A powerful modeling system that integrates climate, crop, hydrology, forest, and economic models for national-level climate-change impact assessments

This methodology combines information on land, water, agro-climatic indicators, crop suitability, and potential yields.

A modeling system that integrates information from climate models, crop simulation models until 2050, and water models linked to a core global, partial equilibrium, multimarket model focused on the agriculture sector

A software application program that comprises dynamic crop growth simulation models for over 42 crops. DSSAT's models simulate growth, development, and yield as a function of soil-plant-atmosphere dynamics.

This 6-year platform (2017-2022) aims to democratize decades of agricultural data to mine information and develop rapid, accurate, and compelling recommendations for farmers, researchers, and policymakers.

A crop-specific water balance model to monitor and predict crop productivity and drought vulnerability during the growing season in drought-prone areas. It can use weather station or satellite data for particular crops in the target regions.

Partnership for Online, map-based platform to provide user-friendly access and interaction with a variety of climate and nonclimate data useful to adaptation planning. Allows for overlays of different datasets, creation of graphs, and customized dashboards.

Tool that allows users to identify suitable crops for a given specific environment, habit of growth, or for a defined use. Can also look up environmental requirements and uses of over 2,300 species.

\section{HOW COULD THIS TOOL BE LEVERAGED FOR} TRANSFORMATION?

Identify crops most vulnerable to climate change.

Understand crop suitability shifts.

Identify alternative crops.

Build capacity among scientists and support stakeholder engagement for long-term decisionmaking.

Inform long-term land use planning where and how production systems might need to shift.

Help evaluate trade-offs of different transformation interventions.

Shed light on where, how, and what transformation interventions might happen.

Provide information on whether market infrastructure exists for particular crops.

Identify most vulnerable crops to climate change.

Understand crop suitability shifts.

Identify alternative crops under different climate scenarios.

Help introduce and promote new CS technologies and innovations at scale.

Understand crop suitability shifts and food security outlooks.

Identify critical areas where transformation might be needed.

Identify critical areas where transformation might be needed.

Help identify potential trade-offs of different transformation interventions by overlaying datasets of interest.

Help identify alternative crops. 


\section{APPENDIX B. INCLUSION OF TRANSFORMATIVE ADAPTATION IN NAPS, NDCS, AND KJWA SUBMISSIONS}

The following table lists the seven entities, in this case all countries, that referenced developing CS systems that could be used for transformative adaptation in either their NAPs, NDCs, or KJWA submissions. The table includes the corresponding excerpts, the document in which they were found, and the type of transformative action it may represent. Out of 196 NDCs, only 3 (approximately 1.5 percent) mentioned the need to establish CS systems that could be used to guide transformative changes. Of the nine NAPS available on UNFCCC NAP Central, three (approximately 33 percent) mentioned the need to establish CS systems to support longerterm, systemic change. Of the 48 KJWA submissions, only 1 (approximately 2 percent) from the Philippines mentioned the need for CS systems that would be considered transformative. Most countries are in the early stages of developing CS, and therefore, only a few countries, such as Burkina Faso, Fiji, and Sri Lanka, show evidence that their CS systems will include longterm climate information for use in adaptation planning. Other examples of actions with high-transformation potential mentioned by countries include harnessing CS capacities for cross-sectoral adaptation planning (Burkina Faso, Uruguay) and equity considerations for projected resettlement of vulnerable groups (Fiji).

This analysis was completed by performing a keyword search for the following terms: climate information service, climate projection, forecast, National Meteorological and Hydrological Services (NMHS), meteorology, hydrology, earth observation, and climatology. These search results were only further analyzed if they were discussed within the context of adaptation. Excerpts were considered falling within the context of transformative adaptation if they reflected this paper's definition of transformative adaptation.
COUNTRY

DOCUMENT

EXCERPT FROM DOCUMENT

HOW IT MAY BE USED FOR TRANSFORMATIVE PLANNING

Countries that are demonstrating early use of CS for long-term planning

Fiji NAP

Fili

Preparation of Guided Urban Growth Management Plans and Guided Strategic Land Development Plans that use zoning and buffer zones to support municipal governance and investment by encouraging settlement and development away from vulnerable areas, based on full use of relevant hazard maps and long-term climate projections

Sri Lanka NAP Area of interest: climate forecasting: short-term and seasonal, long-term projections, communication of climate information; establishing a national research program on climate modeling for long-term climate projections
Harnesses long-term climate projections; decisions around relocation away from vulnerable areas

Use of long-term climate projections

\section{Countries that are building up their CS capacity for long-term planning}

$\begin{array}{ll}\text { Benin NDC } & \text { Reinforce knowledge of the climate system and tools for generating climate and } \\ & \text { hydrological information and forecasting climatic hazards; capacity-building needs: } \\ & \text { use of agro-climatology models (capacity building in agro-climatic risk modeling, } \\ & \text { familiarization with DSSAT software, SARRAH, etc.) } \\ \text { Burkina Faso NAP } & \text { The Mathematical Equation Analysis Laboratory of the University of Ouagadougou, } \\ & \text { with support from the NAPA-BKF-UNDP/Japan project called "Improving Capacities to } \\ & \text { Take Better Account of Climate Change-Related Concerns During the Preparation and } \\ & \text { Implementation of Development Plans, Programmes, and Projects," prepared climate } \\ & \text { forecasts for Burkina Faso up to } 2100 \text { and evaluated the vulnerability of various } \\ \text { development sectors. }\end{array}$

\begin{tabular}{|c|c|c|}
\hline Nicaragua & NDC & $\begin{array}{l}\text { Modernization of the country's hydrometeorological services, which allows } \\
\text { Nicaragua to maintain accurate forecasts and early warning systems }\end{array}$ \\
\hline Philippines & KJWA & $\begin{array}{l}\text { Elements for inclusion: climate information services, particularly for small-scale } \\
\text { farmers, small-scale fishers, coastal communities, and rural women }\end{array}$ \\
\hline Uruguay & NDC & $\begin{array}{l}\text { Development of information systems, climate services, and monitoring programs, } \\
\text { particularly for the environmental, agriculture, and emergency sectors, and } \\
\text { development of early-warning systems to support decision-making }\end{array}$ \\
\hline
\end{tabular}

System-wide modernization of hydrometeorological services Equity considerations for most vulnerable; cross-sectoral Cross-sectoral and systemswide planning for climate modeling

Cross-sectoral and long-term planning and projections looking to 2100 


\section{APPENDIX C. STAGES OF APPLYING CS TO TRANSFORMATIVE ADAPTATION AND OUESTIONS FOR}

PLANNING

This table is based on FAO's planning guidelines for agriculture sector NAPs (Carter et al. 2018) and outlines the stages of planning for applying CS to transformative adaptation in agriculture and related planning questions.

\section{TRANSFORMATIVE STAGE}

Assessing Climate Scenarios

Assessing Impacts and Vulnerabilities

Selecting Adaptation Options

Compiling and Communicating Priorities

\section{TRANSFORMATION QUESTIONS}

Are available CS able to produce high-quality climate-risk and crop and livestock viability models for the long term (more than 10 years into the future)?

Can available CS produce climate-change scenarios that demonstrate how climate conditions are projected to evolve over decades and how these scenarios affect crop and livestock production systems?

Are existing CS adequately focusing on long-term impacts and risks (extreme events and slow onset) for crop and livestock systems?

Are available CS considering long-term impacts and risks to related sectors (e.g., water, cities, forestry, etc.) to inform analysis of trade-offs?

Do existing CS produce information, such as shifts in agro-ecological zones and projected geographic boundaries of crop and livestock production systems, through 2050?

Can available CS analyze the performance of agricultural practices under past and current climate conditions to inform how these practices may be adaptive or maladaptive under projected climate conditions?

Can available CS produce information that demonstrate how climate-change risks to crops and livestock shift over time (through 2050 and beyond), including impacts along the agricultural value chain (e.g., water resources, inputs, processing and storage infrastructure, transportation networks, and markets)?

Can existing CS adequately identify the range of end users, including women, indigenous groups, the extremely poor, and the most vulnerable, and understanding their needs for information, communication channels, and application support?

Are users, including farmers, government, development, and private-sector actors, able to access relevant and actionable CS and apply it in selecting adaptation options over various time frames?

Are users applying short-, medium-, and long-term climate projections and gaining awareness of how incremental adaptation options can be incorporated into longer-term transformative pathways?

Are existing CS designed to address multisectoral tradeoffs as related to agriculture?

Can CS be designed to allow for two-way communication of inputs?

Are adaptation priorities over the short, medium, and longer term adequately informed by available CS?

Do existing CS communicate time frames for tipping points (e.g., temperature and precipitation thresholds) in agricultural systems?

Can available CS anticipate how diverse user information needs may change as climate-change impacts evolve and agricultural systems shift and transform over time?

Are government actors using CS to inform agricultural planning and policy over longer time frames?

Can available CS tailor information, communication channels, and application support to women, indigenous groups, the extremely poor, and other marginalized groups?

Are existing CS designed to address uncertainty and climate variability while ensuring that information is actionable?

Can policymakers access CS in a form that allows for addressing trade-offs that may need to be considered during interministerial coordination and investment prioritization?

Do project designers and implementers have access to CS such that they can justify the long-term climate resilience benefits in project proposals?

Do adaptation funders have access to CS that inform investment portfolios and priorities in the long term? 
Do decision-makers sufficiently understand and value the role of CS in prioritizing adaptation options over short-, medium-, and longer-term time frames?

Are available CS receiving adequate budgets and investment to ensure sustainable information provision to the range of agricultural users over the long term?

Do government agriculture actors at local to national levels have the mandate and ability to use CS in planning and implementation of transformative adaptation?

Do CS inform national and community strategies (e.g., NAPs, NDCs, long-term development plans)?

Can existing CS inform budgetary processes such that investments can be strategically sequenced and compounded for transformative change?

Can available CS be matched with information on other stressors, such as population growth and economic shifts, to best inform long-term agricultural planning and policy? 
REFERENCES

Anderson, G., H. Kootval, D. Kull, J. Clements, G. Fleming, T. Frei, and J. Zillman. 2015. Valuing Weather and Climate: Economic Assessment of Meteorological and Hydrological Services. Geneva: Switzerland: World Meteorological Organisation.

aWhere, Inc. 2020. Website. www.awhere.com. Accessed March 11, 2020.

Bhave, A.G., D. Conway, S. Dessai, and D.A. Stainforth. 2016. "Barriers and Opportunities for Robust Decision Making Approaches to Support Climate Change Adaptation in the Developing World." Climate Risk Management 14: $1-10$.

Bose, Hiren Kumar. 2018, "India Climate Dialogue-Farmers Turn to Millets as a Climate-Smart Crop." September 17. https://indiaclimatedialogue. net/2018/09/17/farmers-turn-to-millets-as-a-climate-smart-crop/. Accessed February 25, 2019.

Braimoh, A.K., F. Ngari, G.A. Obuya, X. Hou, and G. Larson. 2016. "Climate Information Services Providers in Kenya." Report Number 103186. Washington DC: World Bank Group.

Bryan, E., T. Deressa, G.A. Gbetibouo, and C. Ringler. 2009. "Adaptation to Climate Change in Ethiopia and South Africa: Options and Constraints." Environmental Science \& Policy 12 (4): 413-26.

Bullock, R, and A. Tegbaru. 2019. "Women's Agency in Changing Contexts: A Case Study of Innovation Processes in Western Kenya." Geoforum 105: 78-88.

Carter, R., T. Ferdinand, and C. Chan. 2018. "Transforming Agriculture for Climate Resilience: A Framework for Systemic Change." Working Paper. Washington DC: World Resources Institute.

CCAFS (Climate Change, Agriculture, and Food Security). 2016. Annual Report 2016: Power of Partnerships. Wageningen, The Netherlands: CCAFS.

Conforti, P., S. Ahmed, and G. Markova. 2018. Impact of Disasters and Crises on Agriculture and Food Security, 2017. Report. Rome, Italy: Food and Agriculture Organization of the United Nations.

Dessai, S., A. Bhave, C. Birch, D. Conway, L. Garcia-Carreras, J.P. Gosling, N. Mittal et al. 2018. "Building Narratives to Characterise Uncertainty in Regional Climate Change through Expert Elicitation." Environmental Research Letters 13 (7): 074005.

Doss, C.R., and M.L. Morris. 2008. "How Does Gender Affect the Adoption of Agricultural Innovations?" Agricultural Economics 25 (1): 27-39.

European Commission. n.d. "What Is Horizon 2020?" https://ec.europa.eu/ programmes/horizon2020/en/what-horizon-2020. Accessed September 25, 2020.

FA0 (Food and Agriculture Organization of the United Nations). 2015. MOSAICC : A Modelling System for Agricultural Impacts of Climate Change. Rome: FAO.

FA0. 2016. The State of Food and Agriculture 2016 (SOFA): Climate Change, Agriculture and Food Security. https://doi.org/ISBN: 978-92-5-107671-2.

FA0. 2018. Mongolia: Impact of Early Warning Early Action, Protecting the Livelihoods of Herders from a Dzud Winter. Rome: FAO.
Georgeson, L, M. Maslin, and M. Poessinouw. 2017. "Global Disparity in the Supply of Commercial Weather and Climate Information Services." Science Advances 3 (5): 1-10. https://doi.org/10.1126/sciadv.1602632.

Government of Tamil Nadu. 2019. "Climate Change Strategy-Adaptation." In Draft Tamil Nadu State Action Plan on Climate Change 2.0, edited by Department of Environment. Chennai, India: Department of Environment, Government of Tamil Nadu.

Graham, R., H. Ticehurst, B. Leathes, S. Wade, E. Visman, S. Bayley, C. Kane et al. 2015. Scoping, Options, Analysis and Design of a 'Climate Information and Services Programme' for Africa (CIASA). Exeter, UK: Met Office.

Gumucio, T., J. Hansen, S. Huyer, and T. van Huysen. 2018. Gender Responsive Rural Climate Services: A Review of the Literature. Palisades, NY: International Research Institute for Climate and Society.

Gupta, S., P. Sen, and S. Srinivasan. 2014. "Impact of Climate Change on the Indian Economy: Evidence from Food Grain Yields." Climate Change Economics 5 (02): 1450001.

Hansen, J., J. Furlow, L. Goddard, H. Nissan, C. Vaughan, A. Rose, F. Fiondella et al. 2019. "Scaling Climate Services to Enable Effective Adaptation Action." Background Paper. Rotterdam, The Netherlands, and Washington, DC: Global Commission on Adaptation.

Harvey, B., L. Jones, L. Cochrane, and R. Singh. 2019. "The Evolving Landscape of Climate Services in Sub-Saharan Africa: What Roles Have NGOs Played?" Climatic Change 157: 81-98.

Hijioka, Y., E. Lin, J.J. Pereira, R.T. Corlett, X. Cui, G.E. Insarov, R.D. Lasco et al. 2014. "Asia." In Climate Change 2014: Impacts, Adaptation, and Vulnerability. Part B: Regional Aspects. Contribution of Working Group II to the Fifth Assessment Report of the Intergovernmental Panel on Climate Change, edited by V.R. Barros, C.B. Field, D.J. Dokken, M.D. Mastrandrea, K.J. Mach, T.E. Bilir, M. Chatterjee et al. Cambridge, UK, and New York: Cambridge University Press, 1327-70.

Hoegh-Guldberg, O, D. Jacob, M. Taylor, M. Bindi, S. Brown, I. Camilloni, A. Diedhiou et al. 2018. "Impacts of $1.5^{\circ} \mathrm{C}$ Global Warming on Natural and Human Systems." In Global Warming of $1.5^{\circ} \mathrm{C}$. An IPCC Special Report on the Impacts of Global Warming of $1.5^{\circ} \mathrm{C}$ above Pre-industrial Levels and Related Global Greenhouse Gas Emission Pathways, in the Context of Strengthening the Global Response to the Threat of Climate Change, Sustainable Development, and Efforts to Eradicate Poverty, edited by V. Masson-Delmotte, P. Zhai, H.-O. Pörtner, D. Roberts, J. Skea, P.R. Shukla, A. Pirani et al.

Huyer, S., J. Hansen, A. Rose, C. Vaughan, and T. Van Huysen. 2017. What We Know about Gender and Rural Climate Services: Preliminary Findings and Guidance. Wageningen, The Netherlands: CGIAR Research Program on Climate Change, Agriculture and Food Security.

IFAD (International Fund for Agricultural Development). 2014، "Nicaragua: Adapting to Markets and Climate Change Project (NICADAPTA)." Brief. Rome, Italy: International Fund for Agricultural Development.

IPCC (Intergovernmental Panel on Climate Change). 2014. Climate Change 2014: Impacts, Adaptation, and Vulnerability, Part A: Global and Sectoral Aspects. Contribution of Working Group II to the Fifth Assessment Report of the Intergovernmental Panel on Climate Change, edited by R.K. Pachauri and L.A. Meyer. Geneva, Switzerland: IPCC. 
IPCC. 2019. Climate Change and Land: Aan IPCC Special Report on Climate Change, Desertification, Land Degradation, Sustainable Land Management, Food Security, and Greenhouse Gas Fluxes in Terrestrial Ecosystems, edited by P.R. Shukla, J. Skea, E. Calvo Buendia, V. Masson-Delmotte, H.-0. Pörtner, D.C. Roberts, P. Zhai et al. Geneva, Switzerland: IPCC.

Islam, S.N., and J. Winkel. 2017. "Climate Change and Social Inequality." United Nations Department of Economic and Social Affairs (DESA) Working Paper No. 152. https://www.un.org/esa/desa/papers/2017/wp152_2017.pdf.

Jones, R.N., A. Patwardhan, S.J. Cohen, S. Dessai, A. Lammel, R.J. Lempert, M.M.Q. Mirza et al. 2014. "Foundations for Decision Making." In Climate Change 2014: Impacts, Adaptation, and Vulnerability, Part A: Global and Sectoral Aspects. Contribution of Working Group II to the Fifth Assessment Report of the Intergovernmental Panel on Climate Change, edited by R.K. Pachauri and L.A. Meyer. Cambridge, UK, and New York: Cambridge University Press, $195-228$.

Knox, J., T. Hess, A. Daccache, and T. Wheeler. 2012. "Climate Change Impacts on Crop Productivity in Africa and South Asia." Environmental Research Letters 7 (3): 034032.

Michalscheck, M., J.C.J. Groot, G. Fischer, and P. Tittonell. 2020. "Land Use Decisions: By Whom and to Whose Benefit? A Serious Game to Uncover Dynamics in Farm Land Allocation at Household Level in Northern Ghana." Land Use Policy 91: 104325.

Mogelgaard, K., A. Dinshaw, N. Ginoya, M. Gutiérrez, P. Preethan, and J. Waslander. 2018. "From Planning to Action: Mainstreaming Climate Change Adaptation into Development." Working Paper. Washington DC: World Resources Institute.

Nissan, H., L. Goddard, E.C. de Perez, J. Furlow, W. Baethgen, M.C. Thomson, and S.J. Mason. 2019. "On the Use and Misuse of Climate Change Projections in International Development." Wiley Interdisciplinary Reviews:' Climate Change 10 (3): e579.

Perrels, A., T. Frei, F. Espejo Gil, L. Jamin, and A. Thomalla. 2013, "SocioEconomic Benefits of Weather and Climate Services in Europe." Advances in Science and Research 10: 65-70.

PREPData. 2018. (Database.) Precipitation. World Resources Institute. Accessed March 5, 2019.

Rippke, U., J. Ramirez-Villegas, A. Jarvis, S.J. Vermeulen, L. Parker, F. Mer, B. Diekkrügger et al. 2016. "Timescales of Transformational Climate Change Adaptation in Sub-Saharan African Agriculture." Nature Climate Change 6 (6): $605-9$.

Rodrigues, J., J. Thurlow, W. Landman, C. Ringler, and R.D. Robertson. "The Economic Value of Seasonal Forecasts Stochastic Economywide Analysis for East Africa." Discussion Paper. Washington DC: International Food Policy Research Institute.

Rogers, David, and V. Tsirkunov. 2013. Weather and Climate Resilience Effective Preparedness through National Meteorological and Hydrological Services. Washington DC: World Bank. https://openknowledge.worldbank. org/handle/10986/15932.
Singh, C., P. Dorward, and H. Osbahr. 2016. "Developing a Holistic Approach to the Analysis of Farmer Decision-Making: Implications for Adaptation Policy and Practice in Developing Countries." Land Use Policy 59: 329-43. https:// doi.org/10.1016/j.landusepol.2016.06.041.

Singh, C., J. Daron, A. Bazaz, G. Ziervogel, D. Spear, J. Krishnaswamy, M. Zaroug et al. 2018. "The Utility of Weather and Climate Information for Adaptation Decision-Making: Current Uses and Future Prospects in Africa and India." Climate and Development 10 (5): 389-405. https://doi.org/10.1080/17565529. 2017.1318744.

Siregar, P.R., and T.A. Crane. 2011. "Climate Information and Agricultural Practice in Adaptation to Climate Variability: The Case of Climate Field Schools in Indramayu, Indonesia." Culture, Agriculture, Food and Environment 33 (2): 55-69.

Sloan, K., E. Teague, T. Talsma, S. Daniels, C. Bunn, L. Jassogne, and M. Lundy. 2018. "One Size Does Not Fit All: Private-Sector Perspectives on Climate Change, Agriculture and Adaptation." In The Climate-Smart Agriculture Papers, edited by T.S. Rosenstock, A. Nowak, and E. Girvetz, Cham. Switzerland: Springer, 227-33.

Slovic, P., M. Finucane, E. Peters, and D.G. MacGregor. 2002. "The Affect Heuristic." In Intuitive Judgment: Heuristics and Biases, edited by T. Gilovich, D. Griffin, and D. Kahneman. New York: Cambridge University Press.

Sustainable Coffee Challenge. 2019. "Framework." https://www.sustaincoffee. org/framework/. Accessed September 25, 2020.

Tall, A., J. Hansen, A. Jay, B. Campbell, J. Kinyangi, P.K. Aggarwal, and R. Zougmoré. 2014a. Scaling Up Climate Services for Farmers: Mission Possible. Learning from Good Practice in Africa and South Asia. Copenhagen, Denmark: Consultative Group on International Agricultural Research Program on Climate Change, Agriculture, and Food Security.

Tall, A., P. Kristjanson, M. Chaudhury, and S. Mckune. 2014b. "Who Gets the Information? Gender, Power and Equity Considerations in the Design of Climate Services for Farmers." Working Paper. Wageningen, The Netherlands: CGIAR Program on Climate Change, Agriculture and Food Security.

TCS (The Climate Service), 2020. The Climate Service website. IBM. www. theclimateservice.com. Accessed March 2020.

Tye, S., and D. Grinspan. 2020. "Building a Climate-Resilient Future for Costa Rica's Coffee Farming Communities: A Case Study of the Coto Brus Region." Working Paper. Washington DC: World Resources Institute.

UNFCCC (United Nations Framework Convention on Climate Change), 2020. National Adaptation Plans. UNFCCC NAP Central. Database. https://www4. unfccc.int/sites/NAPC/News/Pages/national_adaptation_plans.aspx. Accessed September 25, 2020.

United Nations Human Rights Council. 2019. Climate Change and Poverty. Report of the Special Rapporteur on Extreme Poverty and Human Rights. https://www.ohchr.org/Documents/Issues/Poverty/A_HRC_41_39.pdf.

Usher J., C. Phiri N. Linacre, R. O'Sullivan, and U. Qadir. 2018. Climate Information Services Market Assessment and Business Model Review. Washington, DC: USAID-Supported Assessing Sustainability and Effectiveness of Climate Information Services in Africa Project. 
Vaughan, C., J. Hansen, P. Roudier, P. Watkiss, and E. Carr. 2017. "Evaluating Agricultural Weather and Climate Services in Africa." WIREs Climate Change $10(4): 1-33$.

Vermeulen, S.J., D. Dinesh, S.M. Howden, L. Cramer, and P.K. Thornton. 2018. "Transformation in Practice: A Review of Empirical Cases of Transformational Adaptation in Agriculture under Climate Change." Frontiers in Sustainable Food Systems 2. D0l: 10.3389/fsufs.2018.00065.

WASSAN (Watershed Support Services and Activities Network). 2017. Process Manual for Comprehensive Revival of Millets in the Farming Systems and in Household Consumption (Mission on Millets-Andhra Pradesh). Hyderabad, India: WASSAN.

WFP (World Food Programme). 2016. "Food Security Climate Resilience Facility Supporting Community Resilience-Building before and after Climatic Shocks." FoodSECuRE, http://documents.wfp.org/stellent/ groups/public/documents/communications/wfp279583.pdf? ga $=2.214535189 .368957664 .1513674688-1083946025.1513674688$. Accessed September 25, 2020 .
WMO (World Meteorological Organization). 2014. "Annex: Research, Modelling, and Prediction." In Implementation Plan of the Global Framework for Climate Services. Unedited Report. Geneva, Switzerland: WMO.

WM0. 2015. Conference Report: Conference on the Gender Dimensions of Weather and Climate Services. WMO-No. 1148. Geneva, Switzerland: WMO.

WMO. 2018. "Step-by-Step Guidelines for Establishing a National Framework for Climate Services." WMO-No. 1206. Geneva, Switzerland: WMO.

World Bank. 2017. ICT in Agriculture: Connecting Smallholders to Knowledge, Networks, and Institutions. Washington, DC: World Bank.

WRI (World Resources Institute). 2018. Approach and Scope for Providing Support to Adaptation Activities-Addendum l: The GCF's Approach to Adaptation: Analysis and Implications for the Fund. p.11. https://www. greenclimate.fund/documents/20182/1270184/GCF_B.21_Inf.03_ Add.01_-_Approach_and_scope_for_providing_support_to_ adaptation_activities___Addendum_I_The_GCF_s_approach_ to_adaptation__analysis_and_implications_for_the_Fund.pdf/ d178b4f3-412c-29b6-2530-f7792e2114fb. Accessed September 25, 2020. 
ACKNOWLEDGMENTS

We are pleased to acknowledge our institutional strategic partners that provide core funding to WRI: the Netherlands Ministry of Foreign Affairs, Royal Danish Ministry of Foreign Affairs, and Swedish International Development Cooperation Agency.

This working paper is based on research funded by the Bill \& Melinda Gates Foundation. The findings and conclusions contained within are those of the authors and do not necessarily reflect positions or policies of the Bill \& Melinda Gates Foundation.

\section{ABOUT THE AUTHORS}

Laurie Ashley is an independent Climate Resilience Specialist. Her work focuses on integrating climate risk considerations in decision-making across scales and furthering adaptation and resilience strategies across sectors. Contact: Laurie.Ashley@gmail.com

Rebecca Carter, PhD, is Deputy Director of WRI's Climate Resilience Practice and leads its work on transformative adaptation. She is currently contributing to achievement of the goals of the Food Security and Agriculture Action Track of the Global Commission on Adaptation.

Contact: Rebecca.carter@wri.org

Tyler Ferdinand is an Associate with WRI's Climate Resilience Practice. His research focuses on the systemic shifts that are required at the limits of climate change adaptation, particularly in food and agricultural systems.

Contact: Tyler.ferdinand@wri.org

Richard Choularton is Director of Agriculture and Economic Growth at Tetra Tech. He leads Tetra Tech's international development work on agriculture, food security, and economic growth.

Contact: Richard.Choularton@tetratech.com

Nambi Appadurai, PhD, is Director of WRI India's Climate Resilience Practice. His work focuses on climate policy, climate finance, and governance dimensions of climate change adaptation. He leads the work on mainstreaming adaptation into development at national and sub-national levels in India.

Contact: Nappadurai@wri.org

Namrata Ginoya is a manager of resilience and energy access with WRI India's Energy Team. She works on mainstreaming adaptation into development with a focus on energy access.

Contact: nginoya@wri.org

Parvathi Preethan is a Project Associate with WRI India's Climate Resilience Practice. Her work includes policy research on mainstreaming resilience into development and enhancing readiness to access climate finance in India.

Contact: parvathi.preethan@wri.org
World Resources Institute is a global research organization that turns big ideas into action at the nexus of environment, economic opportunity, and human well-being.

\section{Our Challenge}

Natural resources are at the foundation of economic opportunity and human well-being. But today, we are depleting Earth's resources at rates that are not sustainable, endangering economies and people's lives. People depend on clean water, fertile land, healthy forests, and a stable climate. Livable cities and clean energy are essential for a sustainable planet. We must address these urgent, global challenges this decade.

\section{Our Vision}

We envision an equitable and prosperous planet driven by the wise management of natural resources. We aspire to create a world where the actions of government, business, and communities combine to eliminate poverty and sustain the natural environment for all people.

\section{Our Approach}

COUNT IT

We start with data. We conduct independent research and draw on the latest technology to develop new insights and recommendations. Our rigorous analysis identifies risks, unveils opportunities, and informs smart strategies. We focus our efforts on influential and emerging economies where the future of sustainability will be determined.

CHANGE IT

We use our research to influence government policies, business strategies, and civil society action. We test projects with communities, companies, and government agencies to build a strong evidence base. Then, we work with partners to deliver change on the ground that alleviates poverty and strengthens society. We hold ourselves accountable to ensure our outcomes will be bold and enduring.

SCALE IT

We don't think small. Once tested, we work with partners to adopt and expand our efforts regionally and globally. We engage with decision-makers to carry out our ideas and elevate our impact. We measure success through government and business actions that improve people's lives and sustain a healthy environment.

cacreative (P) Copyright 2020 World Resources Institute. This work is licensed under the Creative Commons Attribution 4.0 International License.

commons (i) To view a copy of the license, visit http://creativecommons.org/licenses/by/4.0/ 